Penser les épidémies depuis la Chine, le Japon et la Corée

\title{
Les épidémies entre « Occident » et « Orient »
}

\section{Florence Bretelle-Establet et Frédéric Keck}

\section{(2) OpenEdition \\ Journals}

Édition électronique

URL : http://journals.openedition.org/extremeorient/327

DOI : 10.4000/extremeorient.327

ISSN : 2108-7105

Éditeur

Presses universitaires de Vincennes

\section{Édition imprimée}

Date de publication : 1 septembre 2014

Pagination : 5-19

ISBN : 978-2-84292-411-9

ISSN : 0754-5010

Référence électronique

Florence Bretelle-Establet et Frédéric Keck, « Les épidémies entre « Occident » et « Orient » », ExtrêmeOrient Extrême-Occident [En ligne], 37 | 2014, mis en ligne le 01 septembre 2014, consulté le 22 septembre 2020. URL : http://journals.openedition.org/extremeorient/327 ; DOI : https://doi.org/ 10.4000/extremeorient.327 


\title{
Les épidémies entre «Occident» et «Orient»
}

\author{
Florence Bretelle-Establet et Frédéric Keck ${ }^{1}$
}

L'histoire des épidémies est révélatrice des «peurs en Occident» ${ }^{2}$. Parmi l'ensemble des menaces diffuses qui pèsent sur l'individu, l'épidémie tranche par son caractère soudain, brutal et collectif: elle se manifeste comme une catastrophe dans la vie quotidienne. On distingue alors de la maladie chronique, qui peut fragiliser l'individu, la maladie infectieuse qui semble affecter le groupe social de l'extérieur ${ }^{3}$. Sa capacité à passer de corps en corps met au défi les conceptions établies de la société, dont elle révèle les vulnérabilités. Lorsque le mal s'empare d'un corps, nul ne sait jusqu'où il s'étendra: les barrières du village ou de la ville ne suffisent pas, les collectifs déjà en place sont débordés. Si l'émotion soulevée par l'épidémie s'apaise d'abord en désignant un coupable du mal pour l'expulser et le détruire, l'enquête vise ensuite à cerner le lieu d'où il part pour le contrôler et l'étudier. L'épidémie est alors l'occasion de cartographier les espaces et les corps qui s'y inscrivent. Aussi la séquence des grandes épidémies raconte-t-elle, pour un historien de l'environnement, les étapes de la transformation de l'Occident: guerres civiles (peste, typhus), conquête du Nouveau Monde (variole), révolution industrielle (choléra, syphilis, tuberculose) ${ }^{4}$.

Le savoir sur les épidémies produit par les sociétés occidentales a été décrit comme marquant le passage de la peur à la maîtrise, de la faute à la maladie, du peuple, affecté uniformément, à la population, considérée dans

1. Nous tenons à remercier la Nippon Koten Kenkyukai, en particulier M. Charles Vacher, pour son soutien à la revue. Notre gratitude va également à Richard Kennedy pour son aide au polissage des textes écrits en anglais par des auteurs non anglophones.

2. Delumeau $1978: 132$.

3. Rosenberg 1992.

4. McNeill 1976. 
ses variabilités ${ }^{5}$. La révolution microbiologique du $\mathrm{XIX}^{\mathrm{e}}$ siècle a découvert sous l'ampleur des contagions collectives les régularités des relations entre les hommes et les microbes. Il n'y avait plus besoin d'invoquer les démons du lieu ou les miasmes de l'environnement, puisqu'on pouvait manipuler les bactéries puis les virus dans un laboratoire ${ }^{6}$. En expliquant l'épidémie par les cassures dans la co-évolution des hommes et des microbes, la microbiologie, à la fin $\mathrm{du} \mathrm{XIX}^{\mathrm{e}}$ siècle, ajoutait ainsi un troisième niveau aux deux autres aspects de la réflexion épidémiologique. Alors que celle-ci décrivait jusque-là l'épidémie comme une crise de surmorbidité (niveau phénoménologique) ou comme une propagation démographique et économique (niveau sociologique), ce troisième niveau ontologique apparut déterminant en dernière instance et clé de la maîtrise des épidémies.

Au milieu du $\mathrm{xx}^{\mathrm{e}}$ siècle, des années 1950 au début des années 1980, on pouvait penser que l'arsenal thérapeutique alors disponible était capable de résoudre la question des épidémies, la découverte, à partir des années 1980, de virus très pathogènes et potentiellement pandémiques (VIH/SIDA, Syndrome respiratoire aigu sévère (SRAS), virus Ebola, H5N1) a mis un terme à cette courte période d'optimisme: «Une nouvelle maladie infectieuse fait son apparition chaque année dans le monde, selon l'Organisation mondiale de la santé (OMS). Et tous les cinq ans, l'humanité subit une crise majeure due à l'émergence ou à la réémergence d'un virus », pouvait écrire une journaliste en décembre $2012^{7}$.

La redécouverte de la menace épidémique, à la fin $\mathrm{du} \mathrm{xx}^{\mathrm{e}}$ siècle, a mis essentiellement l'Asie au cœur de ce diagnostic pessimiste, du fait de son écologie, de sa démographie et de son interdépendance croissante avec le reste du monde. L'Asie orientale, et plus particulièrement la Chine du Sud, fut montrée du doigt lors de l'épidémie de SRAS (2002-2003) qui, avant de se diffuser dans le reste de l'Asie jusqu'au Canada, avec une létalité de $10 \%$, fut qualifiée par un journaliste de Time Asia de «syndrome chinois ${ }^{8}$ ». La grippe pandémique, dont on rappelle souvent qu'elle a fait plus de victimes que la guerre en 1918, est décrite comme une menace permanente dont «l'épicentre» est situé dans le Sud de la Chine ${ }^{9}$ où les oiseaux et les cochons, constituant le «réservoir» pour les mutations des virus Influenza, sont abattus en cas

5. Delaporte 2004 .

6. Winslow 1943.

7. Le Point (dernier accès 28 mars 2014). http://www.lepoint.fr/editos-du-point/annejeanblanc/de-nouvelles-maladies-emergentes-a-redouter-14-11-2012-1528856_57.php

8. Greenfeld 2006.

9. Shortridge et Stuart-Harris 1982. 
de nouvelle épidémie et mis sous étroite surveillance en temps ordinaire ${ }^{10}$. $\mathrm{Si}$ « la menace épidémique nouvelle est présente dans notre quotidien d'une façon disproportionnée au regard de sa rareté statistique et du faible nombre de victimes qu'elle entraîne ${ }^{11}$ », c'est donc qu'elle révèle l'imaginaire d'une globalisation dans laquelle l'Asie joue un rôle essentiel.

Alors que l'Asie orientale, en particulier la Chine, et les pays d'Asie du Sud-Est constituent aujourd'hui les foyers les plus menaçants pour un monde occidental inséré dans un mouvement intense d'échange, les peurs que cette partie du monde suscite sont plus anciennes: au XvIII ${ }^{\mathrm{e}}$ siècle déjà, la variole est soupçonnée par certains médecins européens d'être une maladie asiatique arrivée en Occident ${ }^{12}$. La peste qui éclate à Canton et à Hongkong en 1894 apparaît comme une menace majeure pour la communauté occidentale, et induit par exemple le gouvernement français à renforcer sa présence médicale en Chine du Sud pour éviter que la maladie ne se propage dans la colonie indochinoise, voire en métropole ${ }^{13}$. C'est cette même inquiétude qui conduit les puissances européennes et américaines, au travers d'un Dudgeon (The Diseases of China. Causes, Conditions, and Prevalence, Contrasted with those of Europe, 1877), d'un Maxwell ou d'un Jefferys (The Diseases of China, Including Formosa, and Korea, 1910 et 1929) pour la Chine, d'un Angier pour le Cambodge (Le Cambodge. Géographie médicale, 1901), d'un Rouffiandis pour le Laos (Géographie médicale du Moyen Laos, 1902) ou d'un Gaide pour l'Indochine, à inventorier et cartographier les maladies potentiellement épidémiques de l'Asie, alors en grande partie colonisée par les puissances européennes ${ }^{14}$. Au tournant du $\mathrm{xx}^{\mathrm{e}}$ siècle, comme aujourd'hui, l'Asie fournit également le cadre idéal pour la recherche des causes et des mécanismes de transmission et la mise au point de thérapeutiques. Les expériences menées par Alexandre Yersin sur la peste bubonique sont bien connues. Mais les investigations s'étendent bien au-delà du seul cas de la peste et sont intimement liées au contexte de colonisation. Dans cette époque de grande interrogation médicale, le territoire colonisé en général, et l'Asie en particulier, fournit un

10. Keck 2010.

11. Bourdelais 2003 : 9.

12. Heinrich 2008.

13. Bretelle-Establet 2002.

14. Ces ouvrages développent à la fois un projet cartographique destiné à mettre en évidence des liens de causalité entre épidémies et environnements et, en même temps, une image de l'espace chinois ou indochinois contrastant avec celui de l'Europe. Sur l'usage et les fonctions de ces cartes médicales, $c f$. Hanson, à paraître; MonnaisRousselot 1999. 
cadre de choix à la recherche sur les maladies infectieuses en même temps qu'un terrain d'expérience et d'expérimentation.

En somme, il y a une certaine continuité dans la manière dont le regard occidental appréhende la source des épidémies: l'Asie en est souvent le foyer, qu'il s'agisse de résurgence de maladies épidémiques connues comme la peste ou le choléra, au tournant du $\mathrm{Xx}^{\mathrm{e}}$ siècle, ou qu'il s'agisse de maladies émergentes, comme le SRAS au début du XXI ${ }^{\mathrm{e}}$ siècle. La continuité est aussi visible dans la manière de juger l'approche qu'en font les autorités locales. Les Européens et Américains qui arrivent en Chine à la fin du XIX ${ }^{\mathrm{e}}$ siècle expriment généralement leur consternation devant l'absence de mesures gouvernementales dans la gestion des épidémies, eux qui ont vu, depuis la seconde moitié du $\mathrm{XIX}^{\mathrm{e}}$ siècle, l'essor d'un mouvement médical où la puissance publique s'est donné le droit et même le devoir d'imposer aux individus des règles d'hygiène pour le bien de la collectivité ${ }^{15}$. Aujourd'hui encore, l'épidémie de VIH/Sida, qui ralentit en Occident alors qu'elle progresse en Asie et en Afrique, suscite des discussions sur la résistance des populations et des États à suivre les traitements prescrits par les organisations internationales de santé ${ }^{16}$. Le cas de la pandémie de grippe H1N1 en 2009 montre bien la complexité des relations entre Chine et Occident dans le contrôle des épidémies : après que la directrice de l'Organisation Mondiale de la Santé, Margaret Chan, qui fut directrice de la Santé à Hong Kong entre 1994 et 2003, lança l'alerte sur un nouveau virus, les autorités chinoises, accusées d'avoir sous-estimé le danger du SRAS en 2003, furent accusées par les observateurs occidentaux d'avoir imposé des mesures de quarantaine trop fortes contre le $\mathrm{H} 1 \mathrm{~N} 1{ }^{17}$. Il faut donc renoncer à partir d'un centre de savoir détenteur de la rationalité sur les épidémies pour voir comment celles-ci sont pensées ailleurs.

L'histoire des concepts relatifs aux épidémies et des réponses - politiques, religieuses, médicales - qu'elles suscitent est mieux connue pour l'Europe qu'elle ne l'est pour l'Asie orientale. Ce volume réunit sept contributions traitant d'épidémies qui frappèrent, à différentes époques, la Chine, le Japon et la Corée. Il ne se donne par pour objectif de faire une histoire des épidémies dans cette partie du monde; en étudiant les pratiques politiques, religieuses, économiques que ces épisodes épidémiques suscitent, en scrutant les discours qui les évoquent, tenus dans les milieux médicaux, administratifs ou religieux, il entend plutôt apporter un éclairage sur les manières dont le phénomène

15. Fee et Porter 1992; Guillaume 1996.

16. Micollier 2006; Fassin 2006.

17. Mason 2010. 
épidémique est identifié, compris et combattu dans ce qui est aujourd'hui considéré comme un des principaux foyers d'épidémies.

\section{Les épidémies en Chine, au Japon et en Corée: problèmes de définition}

Mettre au jour les notions qui sous-tendent le phénomène épidémique en différentes parties de l'Asie orientale et le type de réponses qu'il suscite nécessite tout d'abord d'identifier les termes mêmes qui le traduisent. Certes cette question ne se pose pas avec la même acuité pour les différentes époques, pré-moderne et moderne, que ce volume recouvre. S'il semble y avoir un consensus sur les manières dont le phénomène épidémique est compris au $\mathrm{XXI}^{\mathrm{e}}$ siècle, notamment parce qu'il fait l'objet d'une réglementation internationalement reconnue, en revanche la définition des épidémies pose des difficultés plus grandes pour les périodes antérieures.

En Chine, de nombreux termes ont été utilisés dans l'histoire pour désigner des maladies frappant collectivement et de manière spectaculaire ${ }^{18}$. Le caractère $y i$ (qui contient la clé de la maladie et le caractère pour "frapper», et que certains dictionnaires interprètent comme une maladie qui se répand, liuxing) semble le plus couramment utilisé en Chine, en Corée ou au Japon, dans les textes administratifs mais aussi médicaux. Comme le rappelle Marta Hanson dans son étude sur l'évolution du discours médical relatif aux épidémies en Chine, il y a, au XVII ${ }^{\mathrm{e}}$ siècle, une intersection entre le concept «d'épidémies fébriles wenyi » et celui des «maladies chaudes wenbing», ce dernier étant, depuis le $\mathrm{XII}^{\mathrm{e}}$ siècle, associé au sud géographique. Du milieu du XVII jusqu'à la fin du XIX ${ }^{\mathrm{e}}$ siècle, les concepts de «maladies chaudes» et «d'épidémies fébriles» sont donc souvent associés, parfois utilisés l'un pour l'autre, et au centre d'un nouveau discours médical sur l'épidémie: ce discours rejette notamment l'explication classique fondée sur l'idée d'une cosmologie déterminante et lie davantage le phénomène à une anomalie de l'environnement, lui-même perçu comme intrinsèquement lié à une géographie complexe du territoire, capable de produire des corporalités distinctes. En somme, le terme d'épidémie connaît une généalogie singulière en Chine, à partir du XVII siècle : il renvoie moins au peuple (demos) unifié par le mal qu'à l'anomalie d'un environnement avec laquelle celui-ci est désormais unifié ${ }^{19}$. Pourtant, les

18. Fan Xingzhun 1987 : 263-275. Différentes expressions shiyi,jiyi, zaili ont été utilisées mais le terme $y i$, associé aux caractères wen (clé de la maladie) ou wen (clé de l'eau), semble le plus courant.

19. Hanson 2011. 
phénomènes rassemblés sous ce terme ne semblent pas faire l'objet d'un accord unanime. Florence Bretelle-Establet montre dans ce volume que les caractères yi, wenyi, wenbing utilisés dans les sources administratives pour signaler une maladie exceptionnelle et dont les «morts sont innombrables» (sizhe wusuan) donnent lieu à des descriptions de maladies qui, dans les sources médicales, ne sont pas toujours nécessairement liées à des phénomènes collectifs.

Comment les acteurs, qu'ils soient médecins, administratifs ou simples malades, identifient les épidémies est donc une question qui parcourt l'ensemble des contributions de ce volume. Parfois le phénomène épidémique est mis en relation avec une entité nosologique précise - «épidémie rouge», «effondrement en trois jours», «désorganisation soudaine»- en sorte que se pose le problème, classique pour tout historien des maladies, de parvenir à identifier dans une nomenclature biomédicale les termes anciens ${ }^{20}$. À quelle pathologie spécifique rattacher «l'épidémie rouge», décrite ici par Shin Dongwon, qui s'abat sur la Corée à la fin $\mathrm{XVIII}^{\mathrm{e}}$ siècle et à laquelle le roi Jeongjo (r. 1776-1800) répond par des mesures sans précédent? À quel type de pathologies peut-on associer les «désorganisations soudaines» (huoluan), qui peuvent être à l'origine de crises de morbidité ? Si on peut suivre la traduction en Chine du concept de microbe par celui de chong ou vermine ${ }^{21}$, les descriptions symptomatiques, les explications physiopathologiques d'une affection ou encore les ingrédients thérapeutiques prescrits dans les traités médicaux pré-modernes ne sont pas d'un grand secours pour tenter une adéquation avec les entités nosologiques bio-médicales: le regard et le discours médical pré-moderne s'articulent autour de déviances qui ne sont pas toujours significatives aux yeux d'un médecin moderne.

Si tenter un diagnostic a posteriori, sans les examens de laboratoire ou les traces paléo-pathologiques, reste toujours très hasardeux et, à ce titre, ne constitue pas la finalité des recherches de ce volume, il est possible en revanche de suivre la façon dont les phénomènes épidémiques sont perçus, éprouvés, catégorisés, jusqu'à faire l'objet de mesures que l'on qualifierait inadéquatement de «santé publique». En évitant ainsi de projeter les catégories de la santé publique sur des épidémies nettement identifiées, les articles qui suivent retracent le sens du phénomène épidémique à partir des discours et des pratiques des acteurs qui les rencontrent. On retrouve ainsi la dimension imaginaire des épidémies, non comme un obstacle à leur identification précise, mais comme le moteur de leur prise en charge collective.

20. La question a été soulevée par de nombreux historiens, dont Despeux et Obringer 1997 : 20, au sujet de la toux.

21. Andrews 1997. 


\section{Les imaginaires de la menace}

L'épidémie est d'abord une menace pour l'ordre public, qu'elle trouble de ses différents bords. En fragilisant l'ordre social et économique, une maladie entre en corrélation avec d'autres «fièvres", qui peuvent venir de l'intérieur ou de l'extérieur de la société. Cette menace, les contributions de ce volume la présentent sous différents traits.

Si l'histoire européenne fournit de multiples exemples d'épidémies mises sur le compte de l'étranger, les articles de William Johnston ou de Vincent Rollet montrent que le même raisonnement était à l'œuvre dans les sociétés asiatiques. Comme l'épidémie de choléra qui atteint le Japon au XIX ${ }^{e}$ siècle est liée à l'arrivée des vaisseaux européens, les médecins japonais trouvent dans ces mêmes vaisseaux les traités médicaux pour décrire les symptômes : une telle corrélation autorise William Johnston à dire que c'est bien du même choléra que parlent les deux nations en présence. Le choléra, hautement contagieux, est dans un premier temps perçu comme une invasion de l'extérieur, en provenance de Java et de Chine, notamment; au fil du temps, et à mesure que la maladie s'implante au Japon, elle devient aussi une maladie du lieu : füdobyō. La gestion du SRAS à Taiwan, décrite par Vincent Rollet, illustre également cette peur de l'invasion étrangère. Le SRAS fut construit par les autorités taiwanaises comme un problème de sécurité nationale parce qu'il mettait en question les infrastructures vitales de la société, mais aussi parce qu'il arrivait du continent chinois: il fallait se préparer à une nouvelle épidémie comme on se prépare à une attaque militaire. Le SRAS fut décrit par les autorités chinoises comme une pneumonie atypique (feidian), mais sa désignation par l'OMS (Syndrome Respiratoire Aigu Sévère) a pu être associée, dans les articles de République Populaire de Chine, à l'acronyme de la Région Administrative Spéciale de Hong Kong (SAR, Special Administrative Region) et ainsi laisser penser à une épidémie d'origine étrangère.

La menace épidémique peut aussi être mise sur le compte de la désorganisation sociale ou de la décadence des mœurs. Ainsi, Shin Dongwon montre que, dans la Corée du XVIII ${ }^{\mathrm{e}}$ siècle, le non-respect de certains tabous par la population, comme le déménagement des tombes à des moments non propices de l'année, est perçu par le peuple comme la principale cause de l'épidémie rouge hongyeok qui frappe alors le pays. C'est en creux que Florence BretelleEstablet retrouve cette notion: en mangeant de façon équilibrée, en modérant ses passions et son style de vie, on se met à l'abri des miasmes tant redoutés de l'extrême sud de la Chine. Les mouvements de population à l'intérieur du territoire sont également perçus comme des troubles du corps social et des facteurs de propagation des épidémies: en dépit du silence politique des autorités centrales communistes, au cours de la Révolution culturelle étudiée 
par Fan Ka wai, quelques autorités locales mettent en rapport la flambée de méningite et les déplacements des étudiants venus écouter Mao Zedong à Pékin et honorer les lieux sacrés de l'histoire communiste.

Enfin, l'environnement peut aussi constituer la source de l'épidémie. Florence Bretelle-Establet montre que, pour les médecins chinois de la fin de l'empire travaillant dans les régions de l'extrême sud, l'épidémie provient presque toujours d'un environnement pollué dont émane un qi putride et ubiquitaire affectant la collectivité. L'épidémie s'explique parfois par les changements dans l'environnement, et notamment dans les relations entre les hommes et les animaux. Christos Lynteris analyse les débats entre médecins occidentaux sur la peste bubonique en Mandchourie et la synthèse qu'en produit Wu Liande (Wu Lien-teh), médecin chinois formé en Angleterre. En montrant que la peste bubonique est transmise aux humains par les marmottes, ces médecins ne se contentent pas de reprendre un imaginaire de la saleté commune aux hommes et aux animaux, mais posent la question des origines de l'épidémie: pourquoi les chasseurs de marmottes ne sont-ils pas infectés? Pourquoi les coolies qui les tuent pour le marché européen de la fourrure le sont-ils? Y a-t-il un savoir local qui protège de la transmission de l'épidémie par les marmottes? Les spéculations des médecins européens sur le rôle des animaux dans la transmission des épidémies ouvrent de nouvelles possibilités de combinaison, car on peut aussi bien imaginer que les marmottes soient infectées par les cadavres humains qu'elles dévoreraient.

Maladies générées par un cosmos déréglé, des environnements pollués, des divinités courroucées ou encore des envahisseurs étrangers, les épidémies ne laissent pas indifférentes les populations qui en subissent les assauts ni les administrateurs gouvernementaux.

\section{Réponses apportées aux épidémies}

Les réponses à la menace permettent de mesurer l'épreuve que constitue l'épidémie pour une société. En Asie orientale comme ailleurs, la société et ses élites peuvent d'abord recourir au large éventail des pratiques religieuses, visant à restaurer l'ordre bouleversé par l'épidémie. Les fonctionnaires chinois étudiés par Florence Bretelle-Establet ne se contentent pas d'adresser des prières aux divinités orthodoxes des fonctionnaires, les Dieux des Murs et des Fossés, pour éloigner les épidémies meurtrières qui ébranlent le Yunnan au XIX ${ }^{e}$ siècle, mais ils soutiennent aussi les processions populaires destinées à émouvoir les dieux des épidémies. «L'épidémie rouge» analysée par Shin Dongwon dans la Corée de la fin du XVIII ${ }^{\mathrm{e}}$ siècle incite le roi Jeongjo à rétablir, dans le moindre de leurs détails, les rituels aux dieux des épidémies définis 
en 1403 mais rarement respectés depuis. Enfin, l'utilisation d'amulettes bouddhistes est préconisée dans le Japon du milieu du XIX ${ }^{\mathrm{e}}$ siècle pour chasser les démons locaux capables de semer le choléra. À travers ces actions de repentir, ces sacrifices rituels ou ces pratiques apotropaïques violentes, on entre donc en relation, sous différentes modalités, avec les «dieux des épidémies» et leurs acolytes, subalternes, les «démons épidémiques», perçus comme jouant un rôle majeur en Chine, en Corée ou au Japon, dans la propagation des épidémies ${ }^{22}$. La religion n'est pas non plus éloignée du discours des médecins occidentaux du début du $\mathrm{xx}^{\mathrm{e}}$ siècle, même si elle opère à un autre niveau. Les médecins occidentaux analysés par Christos Lynteris spéculent ainsi sur le rôle des pratiques lamaïstes dans la transmission de la peste bubonique. Alors que certains supposent que l'exposition religieuse des cadavres à ciel ouvert favorise la transmission de l'épidémie des humains aux marmottes, d'autres affirment au contraire que l'interdiction de consommer les marmottes limite la propagation - où l'on voit que le religieux est conçu comme une connaissance imparfaite ou tacite de l'environnement.

Si les imaginaires religieux permettent de décrire les réactions populaires aux épidémies, il est difficile de les analyser indépendamment de la mise en scène qui en est donnée par le pouvoir politique. L'épidémie constitue en effet toujours une épreuve pour la souveraineté du pouvoir, mis au défi d'instaurer des mesures visant à redéfinir l'espace sur lequel il s'exerce avec légitimité. La quarantaine, l'isolement, le confinement sont des façons d'exclure l'individu suspect, et ainsi de rétablir une frontière entre le sain et le malade. Comme l'ont montré différents chercheurs, la lèpre ou la variole ont, dans l'histoire chinoise, fait l'objet de telles mesures d'exclusion: dès le $\mathrm{XVI}^{\mathrm{e}}$ siècle, pour la première, et dès la fin du XVII ${ }^{e}$, pour la seconde ${ }^{23}$. Dans la seconde partie du XIX ${ }^{e}$ siècle, l'établissement des concessions étrangères et des ports ouverts a précipité et élargi ces pratiques dans le contexte plus large d'une «hygiène moderne ${ }^{24}$ ». La santé publique apparaît alors comme une façon pour le pouvoir d'affirmer sa souveraineté sur un territoire. Dans ce volume, l'article de Michael Shiyung Liu révèle combien la construction de la santé publique à Taiwan après 1949 ne peut se comprendre sans le contexte de la guerre sino-japonaise, qui a déterminé l'aide américaine apportée au gouvernement nationaliste, à travers un ensemble

22. Schipper 1985; Katz 1995.

23. Leung 2009 : 96-111. Les premiers asiles sont créés à la fin $\mathrm{du}_{\mathrm{XvI}}^{\mathrm{e}}$ siècle, mais la majeure partie date du XVIII ${ }^{\mathrm{e}}$ siècle. Par crainte d'attraper la variole, les manchous qui n'ont pas d'immunité contre la maladie en viennent à dessiner une ville entièrement interdite aux Chinois. (Hanson 2011.)

24. MacPherson 1987; Rogaski 2004. 
d'associations philanthropiques et caritatives. Lutter contre le paludisme à Taiwan dans les années 1950 dans le cadre des institutions de santé mises en place avec les États-Unis, c'est encore faire la guerre à un ennemi, comme l'illustre l'affiche alors en vogue à Taiwan et présentée dans l'article. Vincent Rollet montre combien la «sécuritisation des épidémies » en 2003-2005 permet au gouvernement taiwanais nouvellement élu de gagner en légitimité auprès de son peuple, mais aussi auprès des instances internationales, en se distinguant ostensiblement du gouvernement de la République Populaire de Chine, l'un communiquant ses cas, l'autre les occultant. Dans un contexte international de lutte contre les maladies infectieuses émergentes, la mobilisation de Taiwan contre le SRAS et la grippe aviaire apparaît comme une façon d'affirmer sa souveraineté nationale.

Une autre forme de réponse, destinée à assoir la légitimité de la souveraineté politique, mobilise moins le registre de la guerre et de la sécurité que celui de la surveillance et de la bienveillance. Le savoir épidémiologique a progressivement imposé une dimension d'anticipation dans la gestion des épidémies: il ne s'agit plus seulement de revenir à l'ordre ancien mais de changer les conduites qui ont causé la maladie. « À une protection des frontières de la communauté grâce aux lazarets, aux cordons militaires succède ainsi un ensemble d'efforts orientés vers l'amélioration des équipements sanitaires ainsi que vers une modification des comportements de la population ${ }^{25}$.» Cette dimension d'anticipation, aujourd'hui au cœur des politiques internationales de santé, n'était pas étrangère à la tradition médicale chinoise: le terme de yufang, «prévenir, prévention », est utilisé dans le Classique des Changements (Zhouyi) et nombre de textes anciens, y compris en Corée ou au Japon, soulignent la supériorité de celui qui sait se prémunir contre la maladie avant qu'elle ne soit déclarée. Cette prévention passait cependant par une hygiène individuelle privée composée de pratiques respiratoires, alimentaires, méditatives, et respectueuse de nombreux tabous ${ }^{26}$. William Johnston signale que cette importance accordée à la prévention dans la tradition japonaise a également contribué à la mise en place rapide d'une hygiène moderne et publique à la fin du $\mathrm{XIX}^{\mathrm{e}}$ siècle au Japon. L'anticipation est également au cœur des réponses du gouvernement coréen du roi Jeongjo, à la fin du XVIII ${ }^{\mathrm{e}}$ siècle : à peine «l'épidémie rouge»s'est-elle déclarée que des mesures sont mises en place, en quelques jours seulement, pour limiter la propagation de la maladie et la souffrance générale du peuple; de plus, demande est faite à tout le royaume de faire connaître à la cour les prescriptions les plus efficaces, et de

25. Bourdelais $2003: 12$.

26. Cf. Fan Xingzhun $1953: 8$. Needham et Gu 1962. 
les préparer, par anticipation, avant que ces épidémies cycliques ne terrassent à nouveau le pays; dans la conceptualisation dominante de cette époque, le caractère cyclique et donc prévisible de ces épidémies s'explique par la théorie cosmologique des Cinq Mouvements et des Six souffles (wuyun liuqi). La mise en place de mesures d'urgence et le recours à toutes les forces du pays pour travailler à l'anticipation des prochaines épidémies inscrivent, selon Shin Dongwon, le gouvernement du roi Jeongjo dans une certaine modernité; mais surtout, l'épidémie est ici une épreuve donnant l'occasion au souverain de manifester sa sollicitude à l'égard de son peuple et d'incarner les vertus cardinales qui font l'idéal du bon gouverneur confucéen, ayant à charge la gestion du pays et la santé du peuple (confondues en un seul caractère en chinois $z h i)$.

Quand l'épidémie est conçue comme la propagation anormale d'une maladie naturelle, et non comme la résultante d'une punition divine, la gestion des médicaments est une des épreuves pour la souveraineté: il faut à la fois répondre en urgence par la distribution de produits adaptés, et anticiper par la constitution de stocks. Les sociétés asiatiques pratiquaient la variolisation à titre privé, et ce n'est qu'à partir de 1681 que l'empereur Kangxi l'imposa dans les «enclaves manchoues» aux frontières de l'Empire chinois ${ }^{27}$. L'article de Shin Dongwon montre que l'État coréen, au XVIII ${ }^{\mathrm{e}}$ siècle, publia un plan de rationalisation des médicaments disponibles en cas d'épidémie. Lors de la Révolution culturelle, selon Fan Ka wai, l'épuisement des stocks de sulfadiasine et le manque de masques dans les hôpitaux contribuèrent à la propagation de l'épidémie de méningite cérébrospinale et au constat, par la CIA, de la perte du contrôle de l'épidémie par les autorités communistes centrales. Dans les années 2000, le même type de stockage de médicaments vise à éviter l'effondrement de la croissance économique du fait d'une épidémie de grippe qui entraverait les échanges, et à défendre le statut d'une nation dans le cadre de la «santé globale», comme le souligne Vincent Rollet pour le cas de Taiwan. L'épidémie catalyse tout un jeu complexe de producteurs et de prescripteurs de médicaments dans une logique d'anticipation qui teste la capacité des pouvoirs publics à protéger la population.

\section{Les incertitudes du collectif}

On pourrait penser que l'avènement des théories pastoriennes et de la médecine de laboratoire dans les dernières décennies du XIX ${ }^{\mathrm{e}}$ siècle, qui permirent de comprendre certains des mécanismes liés aux épidémies de maladies

27. Moulin 1996. 
infectieuses, modifie radicalement les différentes figures que prennent la menace épidémique et les réponses qui lui sont apportées. En somme, il devrait $\mathrm{y}$ avoir un avant et un après, ce qui permettrait d'instaurer une coupure pratique dans ce volume entre les contributions portant sur la notion d'épidémie avant le $\mathrm{XIX}^{\mathrm{e}}$ siècle et celles qui traitent de la période contemporaine. Le matériau apporté par ce volume bouscule un tel ordonnancement en montrant que la conceptualisation de la menace épidémique et les différentes formes de réponses qui lui sont apportées ne sont pas fondamentalement différentes d'une période à l'autre et n'obéissent pas seulement aux progrès d'une rationalité scientifique.

L'article de Christos Lynteris montre ainsi que les enquêtes scientifiques menées par des médecins français et russes visant à déterminer l'origine et les mécanismes de la diffusion de la peste dans le Nord de la Chine au tournant du $\mathrm{xx}^{\mathrm{e}}$ siècle s'enchaînent moins comme les étapes d'un progrès linéaire que comme des transformations «épidémio-logiques» autour d'une incertitude fondamentale: que révèle la peste des relations entre les hommes et les marmottes dans un environnement en plein changement? Les articles de Shin Dongwon sur la Corée de la fin du XvIII siècle ou de Fan Ka wai sur la Chine communiste des années 1960 bouleversent l'idée d'une rationalité scientifique appliquée progressivement au contrôle des épidémies. C'est sans connaître le mécanisme de transmission des maladies infectieuses mais en liant le phénomène épidémique à une cosmologie déréglée que le roi coréen Jeongjo, à la fin du XVIII ${ }^{\mathrm{e}}$ siècle, s'active à quadriller la société, à assigner des médecins à chaque quartier, à approvisionner en remèdes coûteux la population la plus démunie. C'est en connaissance des principes de transmission de la méningite cérébro-spinale que le gouvernement communiste poursuit sa politique de mobilisation des masses malgré une épidémie déjà déclarée. L'épidémie véhicule bien plus que l'idée d'une surmorbidité ou d'une surmortalité; quelle que soit la période considérée, elle est une menace pour l'ordre politique, social, économique qui met en branle des réponses allant bien au-delà de la stricte rationalité médicale.

Suivre les réponses à l'épidémie (religieuses, militaires, médicales), c'est donc aussi être attentif aux recompositions du collectif lorsqu'il apparaît à la fois comme cause et comme remède d'une maladie. Les médecins européens ont-ils introduit le choléra ou ont-ils permis aux médecins japonais de le soigner? Les gardes rouges ont-ils propagé ou traité la méningite dans la ferveur religieuse de la Révolution culturelle? Nous avons voulu laisser ouvertes ces questions suscitées par les contributions proposées, car l'un des enseignements des épidémies récentes est la capacité des pouvoirs publics à reconnaître l'incertitude sur les agents infectieux lorsqu'ils se diffusent dans 
un collectif de plus en plus large, et à intégrer un grand nombre d'acteurs pour composer une réponse pertinente.

\section{BibLIOGRAPHIE}

ANDREws Bridie (1997). «Tuberculosis and the Assimilation of Germ Theory in China, 1895-1937». Journal of the History of Medicine and Allied Sciences, vol. 52-1: 114-157.

ARnold David. (1993). Colonizing the Body. State Medicine and Epidemic Disease in Nineteenth Century India. Berkeley, University of California Press.

BouRdelais Patrice (2003). Les Épidémies terrassées. Une histoire de pays riches. Paris, La Martinière.

Bretelle-Establet Florence (2002). La Santé en Chine du Sud (1898-1928). Paris, CNRS Éditions.

Delaporte François (2004). «Épidémie». In Dominique Lecourt (dir.), Dictionnaire de la pensée médicale. Paris, PUF: 418-425.

Delumeau Jean (1978). La Peur en Occident. Paris, Fayard.

DespeuX, Catherine et ObRinger, Frédéric (1997). La Maladie dans la Chine médiévale. La toux. Paris, L'Harmattan.

FAN, Xingzhun 范行準 (1953). Zhongguo yufang yixue sixiang shi 中國預防醫學思 想史 (Histoire de la pensée médicale préventive en Chine). Shanghai, Huadong yiwu shenghuo shi.

FAssin, Didier (2006). Quand les corps se souviennent. Expérience et politiques du sida en Afrique du Sud. Paris, La Découverte.

Fee Elizabeth \& PorTer Dorothy (1992). «Public Health, Preventive Medicine and Professionalization: England and America in the Nineteenth Century». In Andrew Wear (dir.), Medicine in Society: Historical Essays. Cambridge, Cambridge University Press : 249-275.

Guillaume Pierre (1996). Le Rôle social du médecin depuis deux siècles. Paris, Association pour l'étude de l'histoire de la sécurité sociale.

GREENFELD Karl Taro (2006). Le Syndrome chinois. La première grande épidémie du XXI ${ }^{e}$ siècle. Paris, Albin Michel.

Hanson Marta (2011). Speaking of Epidemics. Disease and the Geographic Imagination in Late Imperial China. Londres/New York, Routledge, «Needham Research Institute Series».

Hanson Marta (à paraitre). «Visualizing the Geography of Diseases, 1870s-1920s. An Overview of the Earliest Disease Maps of China».

HeInRICH Larissa (2008). The Afterlife of Images: Translating the Pathological Body Between China and the West. Chapel Hill, Duke University Press.

Katz Paul (1995). Demon Hordes and Burning Boats : The Cult of Marshal Wen in Late Imperial Chekiang. Albany, State University of New York Press. 
KECK Frédéric (2010). «Une sentinelle sanitaire aux frontières du vivant. Les experts de la grippe aviaire à Hong Kong». Terrain, no 54: 26-41.

Leung Angela Ki Che (2009). Leprosy in China: A History. New York, Columbia University Press.

MacPHERSON Kerrie L. (1987). A Wilderness of Marshes. The Origins of Public Health in Shanghai (1843-1893). Oxford, Oxford University Press.

Mason Katherine (2010). «Becoming Modern after SARS. Battling the H1N1 Pandemic and the Politics of Backwardness in China's Pearl River Delta». Behemot. A Journal on Civilisation, $\mathrm{n}^{\circ}$ 3: 8-35.

Mcneill William (1976). Plagues and Peoples. New York, Anchor Books.

Micollier Évelyne (dir.) (2009). «La société chinoise face au SIDA». Perspectives chinoises, $\mathrm{n}^{\circ} 1$.

Monnais-Rousselot Laurence (1999). Médecine et colonisation. L'aventure indochinoise (1860-1939). Paris, CNRS Éditions.

Moulin Anne-Marie (1996). L'Aventure de la vaccination. Paris, Fayard.

Needham Joseph et Lu Gwei-Djen (1962). «Hygiene and Preventive Medicine in Ancient China». Journal of the History of Medicine and Allied Sciences, vol. 17-4: 429-478.

Rogaski Ruth (2004). Hygienic Modernity: Meanings of Health and Disease in TreatyPort China. University of California Press.

Rosenberg Charles (1992). Explaining Epidemics and other studies in the history of medicine. Cambridge, Cambridge University Press.

SCHIPPER Kristofer (1985). "Seigneurs royaux, dieux des épidémies». Archives des Sciences Sociales des Religions, 59, $1: 31-52$.

Shortridge K. F. et Stuart-Harris C.H. (1982). «An Influenza Epicentre?». Lancet, $\mathrm{n}^{\circ}$ ii : $812-813$.

Winslow Charles (1943). The Conquest of Epidemic Diseases. Princeton, Princeton University Press. 
Les épidémies, entre «Occident » et «Orient»

\section{GLOSSAIRE}

$\mathrm{Yi}$ 疫

Liuxing 流行

Wenyi 瘟疫

Wenyi 温疫

Wenbing 温病

sizhe wusuan 死者無算

shiyi 時疫

jiyi 疾疫

zaili 災瘦

huoluan 霍亂

chong 蟲

hongyeok 紅疫

fūdobyō 風土病

feidian 非典

yufang 預防

wuyun liuqi 五運六氣

zhi 治 